\title{
Decoupling of Acetylcholine Influx and Theta Power in the Hippocampus
}

\author{
Calvin K. Young ${ }^{1}$ and Jesse Jackson ${ }^{2}$ \\ ${ }^{1}$ Kavli Institute of Systems Neuroscience and Centre for the Biology of Memory, Medical-Technical Research Centre, Norwegian University of Science and \\ Technology, NO-7489 Trondheim, Norway, and ²Douglas Mental Health University Institute Research Center, McGill University, Montréal, Québec, H4H \\ 1R3, Canada \\ Review of Zhang et al.
}

The importance of acetylcholine (ACh) in higher cognitive functions has been postulated for decades (Sarter et al., 2005). Attention, arousal, novelty detection, and learning all rely on an intact cholinergic system, which arises principally from the nucleus basalis and the medial septum. Traditionally, pharmacological blockade and transmitter-specific lesions have been used to determine the involvement of ACh in neural processes. Later, microdialysis was used to provide a robust measure of the amount of neurotransmitter released in the area of interest with low temporal resolution (i.e., minutes). However, cognitive processes such as attention and arousal, and physiological processes like network rhythms, dynamically change in less than a second. Recent improvements of amperometric recording techniques allow fast, subsecond measurement of extracellular ACh concentration in the brain in vivo (Parikh et al., 2007; Burmeister et al., 2008). With these new tools, old theories regarding the cholinergic system and brain function can now be tested using

Received Nov. 8, 2010; revised Dec. 12, 2010; accepted Dec. 14, 2010.

J.J. was supported by a Natural Sciences and Engineering Research Council of Canada Graduate Scholarship. We thank Drs. Neil McNaughton, Romain Goutagny, Kevin Hascup, and Erin Hascup for their comments on this manuscript.

Correspondence should be addressed to Calvin K. Young, Kavli Institute of Systems Neuroscience and Centre for the Biology of Memory, MedicalTechnical Research Centre, Norwegian University of Science and Technology, N0-7489 Trondheim, Norway. E-mail: calvin.young@ntnu.no.

DOI:10.1523/JNEUROSCI.5862-10.2011

Copyright $\odot 2011$ the authors $\quad 0270-6474 / 11 / 313519-03 \$ 15.00 / 0$ more appropriate physiological time scales $(<1 \mathrm{~s})$.

Hippocampal theta oscillations have been implicated in many cognitive processes such as emotion, reward, learning, and memory (Buzsáki, 2005). In intact animals, hippocampal theta oscillations are known to be modulated by cholinergic, GABAergic, and glutamatergic innervations from the medial septum. The nature of ACh involvement in hippocampal theta oscillations is controversial. Whereas earlier studies postulated that $\mathrm{ACh}$ input to the hippocampus rhythmically pace theta oscillations (Stewart and Fox, 1990), it is clear from more recent studies that septal cholinergic neurons exhibit slow, nonrhythmic activities and thus are unable to rhythmically drive hippocampal theta oscillations (Simon et al., 2006). Lesion studies suggest septal ACh input to the hippocampus is more likely to play a role in the modulation of hippocampal theta power in freely moving animals (Lee et al., 1994) but were demonstrated to be indispensable for hippocampal theta generation under anesthesia (Kramis et al., 1975; Yoder and Pang, 2005). To critically address the role of ACh in hippocampal theta oscillations, a direct demonstration of simultaneous changes in hippocampal field activity and $\mathrm{ACh}$ concentration is necessary. Based on previous work, it would be predicted that under anesthesia (where theta is cholinergic-dependent), ACh concentration should covary with hippocampal theta power. Recent work published in The Jour- nal of Neuroscience by Zhang et al. (2010) used a novel amperometric technique to concurrently record CA1 field potentials and choline concentration in the urethaneanesthetized rat to address the temporal relationships between theta power and $\mathrm{ACh}$ concentration in the hippocampus, furthering our understanding of the role ACh in hippocampal theta oscillations.

The use of in vivo amperometry by Zhang and colleagues (2010) provided the ability to record neurochemical $(<0.5 \mathrm{~Hz})$ and neurophysiological $(>0.5 \mathrm{~Hz})$ signals concurrently by high-pass filtering of an electrical signal obtained by a single sensor (their Fig. 1). The concentration of ACh was assessed by the measurement of choline, the metabolite of ACh. The measurement of choline concentration was made possible by coating the amperometry sensors with choline oxidase and a size-exclusion polymer to limit the signal from potential electroactive interferents. Electrons donated from the oxidation of choline through the $\mathrm{H}_{2} \mathrm{O}_{2}$ intermediate is detected as a current at the recording site held at a positive potential (Bruno et al., 2006). It is important to note that this method of estimating ACh concentration assumes that all ACh released into CA1 is metabolized to choline by the extremely effective acetylcholine esterase (AChE), but does not take into account possible direct choline releases or concentration of preexisting choline. 
By recording from multiple depths within CA1, Zhang and colleagues (2010) localized the largest changes in choline signals to the strata oriens/pyramidale (their Fig. 3), where most of the cholinergic fibers and AChE activity are found (Nyakas et al., 1987). The slow fluctuations in choline signals were correlated to both spontaneously occurring and tail-pinch-induced CA1 theta oscillations with a long latency to peak (their Fig. 2).To address the question of whether ACh release is involved in CA1 theta initiation, Zhang and colleagues (2010) examined the temporal relationships between the choline signal and theta power using the cross-correlation technique. Based on these measures, the time to peak for the choline signal was found to lag behind the time to peak for CA1 theta power by $20-50$ $\mathrm{s}$ (their Fig. 4), prompting the authors to conclude that $\mathrm{ACh}$ is not required for CA1 theta generation. This is an unexpected conclusion because hippocampal theta oscillations in urethane-anesthetized rats have previously been shown to require ACh (Kramis et al., 1975; Yoder and Pang, 2005). The proposal made by Zhang et al. (2010) seems to be based on the assumption that the ACh release in CA1 needs to exhibit the same time course as the simultaneously recorded theta power for ACh to participate in theta generation. This estimate does not take into account the choline already in CA1 because the particular coating technique can only detect changes in choline concentration, not its absolute concentration. If the absolute required $\mathrm{ACh}$ concentration in the CA1 is substantially lower than the peak choline concentration detected, the slight but steady increase of $\mathrm{ACh}$ at the onset of CA1 theta oscillations (their Figs. 2A, $4 A$, $5 F)$ may in fact be sufficient for its contribution to generating $\mathrm{CA} 1$ theta.

Because the dynamics of $\mathrm{ACh}$ release in the hippocampus are predicted to arise from the activity of medial septal cholinergic neurons, Zhang et al. (2010) recorded the activity of septal neurons under the same conditions that hippocampal choline concentration was measured. Previous work has shown that slow-firing septal neurons are most likely to be cholinergic, whereas fast-firing neurons are most likely GABAergic (Stewart and Fox, 1990; Simon et al., 2006). Zhang et al. (2010) found that the time course of the slow-firing septal cells had a temporal profile similar to that of choline concentration, supporting the notion that septal cholinergic neurons are indeed responsible for the changes in choline concentration observed in the postsynaptic CA1 region (their Fig. 5). Fast-spiking neurons (presumably medial septal interneurons) showed a stronger temporal correlation with the emergence of theta oscillations (Zhang et al., 2010, their Fig. 5F), supporting previous claims that medial septal interneurons are involved in hippocampal theta initiation (Hangya et al., 2009). Intriguingly, it is clearly shown in Zhang et al.'s (2010) Figure 5, A, C, F, that the putative medial septal cholinergic neurons, as a population, do in fact increase their firing rate at the onset of CA1 theta oscillations with firing rates rapidly ramping above baseline activity (their Fig. 5C). This contrasts with recorded CA1 choline concentration (their Fig. $5 F$ ), suggesting decoupling between putative septal cholinergic neuron firing rate and CA1 choline concentration around CA1 theta onset. It may be possible that, because Zhang et al. (2010) were detecting choline, the initial ACh increase in the CA1 was masked by a high proportion of receptor-bound $\mathrm{ACh}$ and the relatively low $\mathrm{AChE}$ activity in the CA1 (Perrier et al., 2003) and fast choline uptake. Saturation of receptorbound ACh and choline uptake may eventually result in the subsequent increased temporal coupling between medial septal cholinergic activity and choline concentration in the CA1 (Zhang et al., 2010, their Fig. 5F). Direct measurements of ACh concurrent with choline (Burmeister et al., 2008) may clarify the nature of observed decoupling between choline concentration and putative medial septal cholinergic neuron firing rate.

Although it remains unclear whether $\mathrm{ACh}$ is necessary to initiate hippocampal theta in urethane-anesthetized animals, it is clear that the choline concentration in CA1 is decoupled from theta power recorded from the same region. What then, may be the functional significance be of slow ACh transients reported by Zhang et al. (2010) if they are not related to the generation of hippocampal theta or the modulation of its power? In freely moving animals, it is thought that the presentation of novel stimuli elicits high hippocampal ACh to suppress excitatory intrahippocampal pathways (within CA3 and between CA3 and $\mathrm{CA} 1$ ) and facilitate entorhinal cortex inputs to encourage encoding, whereas low ACh may promote retrieval by releasing CA1/CA3 inhibition (Hasselmo, 2006). Therefore, phasic hippocampal ACh transients may play a major role in coordinating memory encoding and retrieval states. With the techniques described by Zhang et al. (2010), it is possible to directly examine how changes in ACh concentration and hippocampal network activity may vary according to behavior, thereby providing insights to the role of ACh in hippocampal function.
In conclusion, Zhang et al. (2010) provide important insights regarding changes in hippocampal ACh concentrations during the initiation and termination of CA1 theta rhythms in urethane-anesthetized rats. Using improved amperometric recording technology, the authors simultaneously measured local field potentials and estimated ACh concentration with high temporal resolution. Although the time and rate of change of CA1 choline concentration and theta power were not strongly correlated, it remains unclear whether choline measurements used by Zhang et al. (2010) are indeed representative of CA1 ACh concentration, and how much ACh release by septal cholinergic neurons may be required for hippocampal theta generation under urethane anesthesia. Further research should determine how ACh transients are regulated, as well as determining the biological significance of the observed slowly ramping ACh release on hippocampal circuitry. These electrochemical techniques should pave the way for future studies of hippocampal ACh function in freely moving animals. Therefore, Zhang et al. (2010) provide an important first step into a new age of experiments to reevaluate the complex relationship between $\mathrm{ACh}$ and memory systems.

\section{References}

Bruno JP, Gash C, Martin B, Zmarowski A, Pomerleau F, Burmeister J, Huettl P, Gerhardt GA (2006) Second-by-second measurement of acetylcholine release in prefrontal cortex. Eur J Neurosci 24:2749-2757.

Burmeister JJ, Pomerleau F, Huettl P, Gash CR, Werner CE, Bruno JP, Gerhardt GA (2008) Ceramic-based multisite microelectrode arrays for simultaneous measures of choline and acetylcholine in CNS. Biosens Bioelectron 23:1382-1389.

Buzsáki G (2005) Theta rhythm of navigation: link between path integration and landmark navigation, episodic and semantic memory. Hippocampus 15:827-840.

Hangya B, Borhegyi Z, Szilágyi N, Freund TF, Varga V (2009) GABAergic neurons of the medial septum lead the hippocampal network during theta activity. J Neurosci 29:8094-8102.

Hasselmo ME (2006) The role of acetylcholine in learning and memory. Curr Opin Neurobiol 16:710-715.

Kramis R, Vanderwolf CH, Bland BH (1975) Two types of hippocampal rhythmical slow activity in both the rabbit and the rat: relations to behavior and effects of atropine, diethyl ether, urethane, and pentobarbital. Exp Neurol 49:58-85.

Lee MG, Chrobak JJ, Sik A, Wiley RG, Buzsáki G (1994) Hippocampal theta activity following selective lesion of the septal cholinergic system. Neuroscience 62:1033-1047.

Nyakas C, Luiten PGM, Spencer DG, Traber J (1987) Detailed projection patterns of septal and diagonal band efferents to the hippocam- 
pus in the rat with emphasis on innervation of CA1 and dentate gyrus. Brain Res Bull 18: 533-545.

Parikh V, Kozak R, Martinez V, Sarter M (2007) Prefrontal acetylcholine release controls cue detection on multiple timescales. Neuron 56:141-154.

Perrier NA, Khérif S, Perrier AL, Dumas S, Mallet J, Massoulié J (2003) Expression of PRiMA in the mouse brain: membrane anchoring and accumulation of 'tailed' acetylcholinesterase. Eur J Neurosci 18:1837-1847.
Sarter M, Hasselmo ME, Bruno JP, Givens B (2005) Unraveling the attentional functions of cortical cholinergic inputs: interactions between signal-driven and cognitive modulation of signal detection. Brain Res Brain Res Rev 48:98-111.

Simon AP, Poindessous-Jazat F, Dutar P, Epelbaum J, Bassant MH (2006) Firing properties of anatomically identified neurons in the medial septum of anesthetized and unanesthetized restrained rats. J Neurosci 26:9038-9046.
Stewart M, Fox SE (1990) Do septal neurons pace the hippocampal theta rhythm? Trends Neurosci 13:163-168.

Yoder RM, Pang KC (2005) Involvement of GABAergic and cholinergic medial septal neurons in hippocampal theta rhythm. Hippocampus 15:381-392.

Zhang H, Lin SC, Nicolelis MA (2010) Spatiotemporal coupling between hippocampal acetylcholine release and theta oscillations in vivo. J Neurosci 30:13431-13440. 